\title{
Advances in Single-optic Accommodating Intraocular Lenses
}

\author{
John A Hovanesian, MD
}

Clinical Instructor, University of California, Los Angeles Jules Stein Eye Institute, and Private Practice, Harvard Eye Associates, Laguna Hills, California

\begin{abstract}
This article reviews the evolution of single-optic accommodating intraocular lenses (IOLS) from the development of the first commercially available model, the Crystalens AT-45, through to newer models of these presbyopia correcting IOLS, including the Crystalens AO, the Lenstec TetraFlex, the Human Optics 1-CU, and the Tekia Tek-Clear. Current clinical data for all lenses are reviewed, as well as the importance of a thorough pre-operative work-up in order to ensure success with these lenses.
\end{abstract}

\section{Keywords}

Accommodating intraocular lenses (IOLS), presbyopia, baby boomers, quality of vision

Disclosure: John A Hovanesian, MD, is a consultant to Bausch \& Lomb and Abbott Medical Optics.

Received: September 15, 2010 Accepted: February 22, 2011 Citation: US Ophthalmic Review, 2011;4(1):49-51 DOI: 10.17925/USOR.2011.04.01.49

Correspondence: John A Hovanesian, MD, 24401 Calle De La Louisa, Suite 300, Laguna Hills, California 92653. E: DrHovanesian@harvardeye.com

The 'baby boomers', those born between 1946 and 1964, are now in their 60s, 50s, and late 40s. Unlike previous generations, baby boomers view the world with a future-forward perspective. According to a report published in January 2010, baby boomers feel younger than they actually are and fully expect that medical breakthroughs will allow them to live past 100 . However, they are not completely satisfied with their lives: virtually all members of this generation want to make substantial life changes that include taking better care of their physical health. Hand-in-hand with this fact is that baby boomers promise to wield considerable buying power. ${ }^{1}$ These are certainly interesting facts, but how do they relate to advancements in single-optic accommodating intraocular lenses (IOLS)? Quite simply, for this generation, the handicap of presbyopia is not an acceptable situation. Furthermore, after cataract surgery, this population increasingly expects that they can reduce or eliminate the need for glasses. For a growing number of baby boomers, accommodating IOLS represent an effective choice. Clinical studies have demonstrated that accommodating IOLS can reduce or eliminate the need for glasses following implantation, while providing a good range of near, intermediate, and distance vision. Finally, because the optic is a either a spherical or aspheric monofocal, patients do not experience the side effects seen with multifocal IOLs, such as glare, halos, and loss of contrast sensitivity. ${ }^{2}$

Although the use of accommodating IOLs is on the rise today, the story of these IOLs actually dates back approximately 20 years to when ophthalmologist Stuart Cumming began to notice that patients implanted with a $10.5 \mathrm{~mm}$ plate haptic IOL could read even though they were close to emmetropia. When Cumming refracted these patients, even if they were best-corrected for distance, the patients were able to read at $\mathrm{J} 3$ or better under dim light. Slit-lamp examinations in these patients found that the plate haptic lenses had a tendency to vault more posteriorly than was observed with a three-piece IOL. To confirm these observations, an ultrasound study was performed and found that the plate haptic lenses consistently moved to the posterior part of the capsular bag and compressed the vitreous $50 \%$ of the time when the patients accommodated to read.

The ultrasound study found that the most anterior location was only $0.77 \mathrm{~mm}$ in front of the original location of the posterior capsule. By comparison, when three-piece IOLs were looked at, this lens design reduced the vitreous cavity only $20 \%$ of the time and lengthened it by as much as $2.17 \mathrm{~mm}$. As a result of this work, Cumming began to develop a new lens that was designed to increase accommodation. The first prototype was implanted in 1991, with the design being modified as cumming and his colleagues worked to achieve the ideal optic/haptic combination. Thirteen years later, in 2003, the Crystalens AT-45 was approved for use in the US by the US Food and Drug Administration (FDA) and a new era in IOL surgery and presbyopia correction had begun.

The main concept of the Crystalens IOL was to make the optic smaller than standard IOLs while the haptics were designed to be longer. The optic was $4.5 \mathrm{~mm}$ for the original AT-45 model. The reason for reducing the optic size was to create a greater range of travel for a given angle of deflection. With this design, the Crystalens is intended to have two mechanisms of action: forward axial movement of the lens and optic arching. When implanted in a posterior vaulted position within the capsular bag, the accommodative process causes the ciliary muscles to contract, displacing the vitreous mass, and causing forward motion of the Crystalens. ${ }^{3}$

\section{Available Single-optic Accommodating Intraocular Lenses}

Since the commercial introduction of the crystalens, other manufacturers have introduced single-optic accommodating IOLs. Here, we will briefly 


\section{Figure 1: A Simulation of the Decentration Effect}

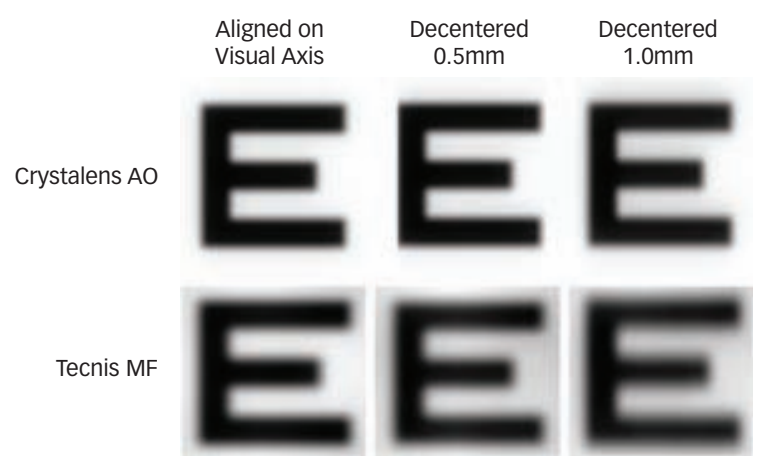

A ray-trace program was used to simulate the effect of decentration on the modulation transfer function of the aspherically neutral Crystalens AO lens and the negative spherical aberration (SA) Tecnis Multifocal (MF) lens through a 3mm pupil. Performance degradation was greater with decentration for the negative SA lens. Astigmatism and coma were the primary asymmetrical higher-order aberrations induced by the lens with negative asphericity.

Figure 2: The Benefit of Crystalens AO with Intraocular Lenses (IOLS) Compared with Multifocal IOLs

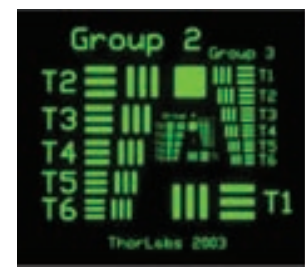

Crystalens AO

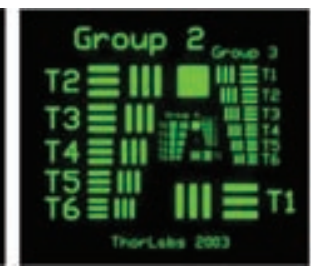

ReSTOR +3

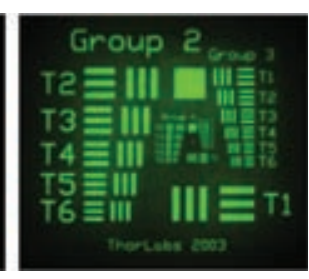

Tecnis MF
Photos of US Air Force Resolving Power Targets through Crystalens AO accommodating IOL, ReSTOR +3 Multifocal IOL, and Tecnis Multifocal (MF) IOL demonstrating the improved clarity of the image through the Crystalens AO accommodating intraocular lenses (IOLS) compared with either of the multifocal IOLS.

review the current models offered by manufacturers, as well as the features and benefits. However, at the time of writing, only one accommodating IOL is commercially available in the US.

\section{Crystalens AO (Bausch + Lomb)}

This is the fifth generation of this silicone, one-piece accommodating IOL to be commercially available in the US and the rest of the world. The AO combines the clinically proven accommodating ability of the Crystalens with the aberration-neutral optic first available with the SoFlex and Akreos IOL platforms. The design is similar to the Crystalens $5-0$ with a $5 \mathrm{~mm}$ optic and an overall length of 11.5mm (17-33D) and 12mm (10-16.75D). The benefits of an aspheric optic include better vision quality relative to standard spherical IOLs as well as more predictable and repeatable refractive outcomes (see Figure 1). ${ }^{4.5}$ In addition, because the AO has consistent power across the entire surface of the optic, the optical performance of the crystalens is generally unaffected by pupil size, mild tilt, or decentration of the optic. This makes the AO more forgiving, particularly when compared with the previous version of the Crystalens, the HD.

\section{The Tetraflex (LensTec)}

This hydrophilic acrylic lens has a $5.5 \mathrm{~mm}$ optic with an overall length of $11.5 \mathrm{~mm}$ and is available in $0.2 \mathrm{D}$ steps. The Tetraflex is available outside the US and is currently undergoing review by the FDA for pre-market approval. The lens has a $5^{\circ}$ anterior vault that is designed to enhance movement during ciliary muscle contraction.

\section{Akkommodative 1-CU (Human Optics)}

This one-piece accommodative IOL is a hydrophilic acrylic with an optic of $5.5 \mathrm{~mm}$ and an overall diameter of $9.8 \mathrm{~mm}$. There is little information regarding the proposed mechanism of action of this $\mathrm{IOL}$, although a 2006 article indicates a similar concept to that of the crystalens: forward movement of the IOL as the ciliary muscles contract. ${ }^{6}$

\section{Tek-Clear Accommodative Intraocular Lens (Tekia)}

This one-piece hydrophilic acrylic lens has a $5.5 \mathrm{~mm}$ optic with an overall length of $10-11 \mathrm{~mm}$, depending on the IOL power. According to the manufacturer, the design of the Tek-Clear utilizes a 'bending-beam' approach intended to optimize the IOL movement as the ciliary muscle contracts and relaxes during the accommodating process. The lens has a square edge, which the company indicates is designed to reduce posterior capsule opacification in order to maintain capsular bag flexibility.

\section{Clinical Results}

As it is the only single-piece accommodative IOL approved in the US, the published clinical results on the crystalens IOL are more extensive than the other available lenses. For the purposes of this article, we will provide a brief review of the most recently available clinical data for each lens.

\section{Crystalens AO}

As part of the initial commercial introduction of this lens, the manufacturer conducted feasibility studies. The results reported by Bausch + Lomb demonstrated that $100 \%$ of patients implanted with the Crystalens AO had uncorrected binocular visual acuities at near, intermediate, and distance vision of J3 and 20/40 or better. ${ }^{7}$ One of the questions that has lingered about single-optic accommodative IOLs has centered on performance over a longer period of time as the capsular bag becomes more fibrotic and, in theory, limits the movement of the IOL. Long-term data on the original Crystalens, ${ }^{8}$ the AT-45, dispel this notion by demonstrating that, seven years following implantation, patients maintained good near, intermediate, and distance vision. In fact, $98 \%$ of patients could read J3 or better at seven years post operative, which actually was an improvement over the one-year results, and 96\% had a binocular uncorrected distance visual acuity of 20/32 or better at seven years.

\section{Tetraflex}

The one-year FDA clinical study results on the Tetraflex were published at the end of 2009. A total of 255 patients underwent implantation of the Tetraflex lens as part of the study, with 239 patients included in the one-year follow-up. In the report, $96 \%$ of Tetraflex patients reported never wearing glasses for distance compared with $80 \%$ of control (monofocal) patients $(p<0.001)$. Seventy-five percent of the Tetraflex patients reported near spectacle wear either never or only occasionally for small print and/or dim light compared with $46 \%$ of control patients $(p<0.001)$. Spectacle independence, as measured by the proportion of patients with uncorrected distance visual acuity of 20/25 or better and various degrees of uncorrected near visual acuity, was also significantly better $(p<0.001)$, as was distance-corrected near visual acuity $(p<0.001)$, compared with the monofocal control eyes. ${ }^{9}$ 


\section{Akkommodative 1-CU}

The most recent clinical data on this accommodative IOL were published in August 2010 in the American Journal of Ophthalmology. In this non-randomized, prospective clinical study, the 1-CU was compared with three other types of IOL: a refractive multifocal IOL (ReZoom, Abbott Medical Optics), a diffractive multifocal IOL (Tecnis, AMO), and a monofocal IOL (Acrysof, Alcon).

This study enrolled 87 patients (87 eyes) with 24 patients implanted with the monofocal (group 1), 21 patients had the accommodating IOL (group 2), 22 patients had the diffractive multifocal (group 3), and 20 patients had the refractive multifocal (group 4). The patients were followed for 18 months and monitored for subjective refractions, monocular and binocular distance, intermediate and near uncorrected visual acuities, monocular distance and near best-corrected visual acuities, monocular distance-corrected intermediate and near visual acuities, stereopsis, visual complaints, and spectacle dependency.

The results found that there was no difference between the near and distance best-corrected visual acuity in all groups, while there was also no difference in the intermediate visual acuities in groups 2,3 , and 4 $(p<0.05)$. The number of patients with better stereoscopic function, spectacle independence, and complaints of halo in groups 3 and 4 was significantly higher than in other groups $(p<0.05)$. Based on these results, the authors concluded that the multifocal IOL patients had better visua function compared with the accommodative IOL (Akkommodative 1-CU) and monofocal IOL groups. ${ }^{10}$

\section{The Importance of Quality of Vision}

With ever-increasing numbers of cataract patients opting for presbyopiacorrecting IOLS, more scrutiny will be placed on the visual outcomes and quality of vision provided by these lenses. As mentioned in this article's introduction, the visual trade-offs experienced by patients implanted with multifocal IOLs have been well documented. ${ }^{2}$ On the other hand, none of the matters, such as glare, halos, or reduced contrast sensitivity, have been seen in patients implanted with accommodative IOLS. This is attributable to the moving single point of focus in these lenses. Or, in the case of the crystalens $\mathrm{AO}$, an aberration-neutral, aspheric optic. This design should provide better quality of vision, with no reduction in contrast sensitivity following surgery."

The Crystalens AO is designed so that the aspheric anterior and posterior surfaces create no spherical aberration. In addition, due to the aberration neutrality of the optic, this IOL is suitable for all patient types; and, whereas visual performance of multifocal IOLS can be compromised by optical misalignment or pupil decentration, the performance of the Crystalens AO is not impacted by less-than-optimal conditions. What this also means is that when patients are implanted with this single-optic accommodating IOL, 100\% of available light rays are available at all distances-so patients see near, intermediate, and at distance with equal clarity as the IOL moves forward and changes its radius of curvature as a patient accommodates at near or performs a visual task at a greater distance.

When the performance of a single-optic accommodative IOL is compared with multifocal IOLs, the advantages of this approach for correcting presbyopia become clear. In a study of modulation transfer function (MTF) using a $3 \mathrm{~mm}$ aperture, the crystalens $\mathrm{AO}$ demonstrated enhanced improved MTF performance over two multifocal lenses (AcrySof ReSTOR ${ }^{\circledR}$ and ReSTOR ${ }^{\circledR}$ Aspheric). In a separate study, using Air Force target photos to compare quality of vision also demonstrated better quality of vision with this accommodative IOL compared with two types of multifocal IOLS (ResTOR and Tecnis) (see Figure 2).

The use of these two tests-modular transfer function and the Air Force target tests-raises other points that are important when discussing any type of presbyopia-correcting IOL: ensuring a quality visual outcome using careful pre-operative planning, as well as in the post-operative assessment. In a recently published article, Pepose makes the point that the use of presbyopia-correcting IOLs requires more than standard Snellen acuity assessment. ${ }^{12}$ Pre-operatively, this means careful biometry and IOL calculations, as well as assessing what Pepose calls the "total ocular characteristics" before operating. This includes corneal topography and careful slit-lamp examinations to identify any corneal irregularities. ${ }^{12}$ The pre-operative work should also ensure that any ocular surface disease is diagnosed and addressed prior to surgery. Lastly, if there is more than 0.75 diopters of astigmatism, than either limbal-relaxing incisions or astigmatic LASIK should be planned in order to ensure the best possible outcome.

After surgery, the use of low- and high-contrast visual acuity provides a more accurate picture of a patient's vision and can better pinpoint any issues should a patient refract to $20 / 20$, but express dissatisfaction with the visual result.

Our clinical experience with the Crystalens $\mathrm{AO}$, as well as the earlier models of this single-optic accommodating IOL, has been positive due to the fact that we are able to reduce or eliminate the need for glasses, particularly for reading, following cataract surgery. The added benefit of providing patients with a good range of vision, without any visual trade-offs, is an important reason for encouraging use of these presbyopiacorrecting IOLS.
1. Attitudes and Expectations. January 2010. Website of the American Association for Homes and Services for the Aging.

2. Leyland $M$, Pringle $E$, Multifocal versus monofocal intraocula lenses after cataract extraction, Cochrane Database Syst Rev 2006; (4):CD003169

3. Cumming JS, Kammann J, Experience with an accommodating IOL (letter), J Cataract Refract Surg, 1996;22:1001

4. Laboratory data on file at Bausch \& Lomb Incorporated.

5. Altmann GE, Nichamin LD, Lane SS, et al. Optical performance of 3 intraocular lens designs in the presence of decentration, J Cataract Refract Surg, 2005:31(3):574-85.
6. Wolffsohn JS, Hunt OA, Naroo S, et al., Objective accommodative amplitude and dynamics with the $1 \mathrm{CU}$ accommodative intraocular lens, Invest ophthalmol Vis Sci, 2006:47(3):1230-5.

7. Data on file, Surgivision ${ }^{\circledR}$ DataLink

8. Colvard D, Doane JF, Kandavel R, Near, Intermediate, and Distance Vision 7 Years After Accommodating IOL Implantation. Annual Symposium of the ASCRS Abstract. 2011.

9. Sanders DR, Sanders ML, Tetraflex Presbyopic IOL Study Group. US FDA clinical trial of the tetraflex potentially accommodating IOL: comparison to concurrent agematched monofocal controls, J Refract Surg,
2010;26(10):723-30.

10. Mesci C, Erbil HH, Olgun A, Yaylali SA, Visual performances with monofocal, accommodating, and multifocal intraocular lenses in patients with unilateral cataract, Am I Ophthalmol, 2010;150(5):609-18.

11. Pepose JS, Qazi MA, Edwards KH, et al., Comparison of contrast sensitivity, depth of field and ocular wavefront aberrations in eyes with an $1 \mathrm{OL}$ with zero versus positive spherical aberration, Graefes Arch Clin Exp Ophthalmol, 2009:247(7):965-73

12. Pepose JS, The impact of high-quality vision in patients with accommodating IOLs, Advanced Ocular Care, 2010;21-4. 